\title{
Effects of Different Artificial Agings on Structure and Properties of Whatman Paper Samples
}

\author{
M. Cocca, ${ }^{1}$ L. D’Arienzo, $^{2}$ and L. D’Orazio ${ }^{1}$ \\ ${ }^{1}$ Istituto di Chimica e Tecnologia dei Polimeri del CNR Via Campi Flegrei, 34-80078 Pozzuoli (NA), Italy \\ ${ }^{2}$ Dipartimento di Ingegneria Chimica e Alimentare, Università di Salerno, Via Ponte don Melillo, 84087 Fisciano (SA), Italy
}

Correspondence should be addressed to L. D’Orazio, loredana.dorazio@ictp.cnr.it

Received 20 April 2011; Accepted 20 May 2011

Academic Editors: X. Colom, J. Greener, M. Lebyodkin, and N. Uekawa

Copyright ( $\odot 2011$ M. Cocca et al. This is an open access article distributed under the Creative Commons Attribution License, which permits unrestricted use, distribution, and reproduction in any medium, provided the original work is properly cited.

\begin{abstract}
With the aim at controlling cellulose degradation phenomena, the natural aging of Whatman paper samples was simulated through different artificial aging processes: thermal oxidation in air at constant temperature, photo-oxidation under Xenon arc lamp, accelerating ageing in climatic chamber, and chemical oxidation with sodium methaperiodate. The cellulose degradation was studied by means of viscosimetric, Fourier transform infrared spectroscopy (FTIR), and wide angle X-ray scattering (WAXS) techniques together with thermogravimetric (TGA), mechanical, and optical analyses. All the treatments carried out were found to significantly modify paper structure and properties, the extent of the deterioration effects depending upon the aging kind. Direct correlations were, nevertheless, assessed among level of cellulose molecular degradation, formation of carboxyl, and/or carbonyl groups and paper strain at break.
\end{abstract}

\section{Introduction}

Paper is one of the oldest and widely used man-made materials. It is mostly made from cellulose and water with small amounts of organic, inorganic, and, possibly, dye additives [1]. While in earlier hand-made and modern highquality machine papers only cellulose fibers are present [2], in low-quality paper, the cellulose is embedded in an hemicellulose and lignin matrix.

The main alterations of cellulose-based materials are induced by photodegradation, acid hydrolysis, oxidation, and biodegradation. Since these phenomena are all related to each other, a full analysis of the material "cellulose" is quite complex.

The oxidation of cellulose may be brought about by a wide range of oxidizing agents [3]. Molecular oxygen can oxidize cellulose, the rate of the attack being greatly accelerate by alkaline conditions and high temperatures. All forms of oxidative attack result in a modification of the cellulose structure, a shortening of the cellulose chain, and a weakening of the cellulose fibres.

It is well known that cellulosic materials are damaged by prolonged exposure to light [4]. The visible consequences are some degree of discoloration accompanied by a general loss in strength. Two distinct mechanisms are to be considered: in the former, the light energy is the principal factor responsible for the cellulose degradation, but in the latter, the type of impurities and the presence of moisture and oxygen contribute to the attack. The exposition to high-energy radiation in the ultraviolet range (photolysis) produces the scission of the chemical bonds in the cellulose chain [5]. At last, photosensitization is another degradative phenomenon correlated to the exposition to the light.

Presently, a great scientific interest is focused on the study of the cellulose degradation mechanisms in order to achieve a deeper understanding of its degradation phenomena, the final goal being to improve the existing methods of conservation and restoration of cultural heritage consisting of cellulose-based materials [6-8].

Starting from this need, with the specific object of obtaining paper samples with controlled degradation levels, suitable to be used as model samples, accelerating ageing cycles were set up reproducing different degradation processes undergone by ancient paper items. In particular, in this work artificial ageing procedures of thermal oxidation, photo-oxidation under Xenon arc lamp, accelerating ageing 
in climatic chamber and chemical oxidation were performed on Whatman paper samples.

The degradation level of cellulose for each aging procedure was evaluated by measuring the cellulose intrinsic viscosity, by Fourier transform infrared spectroscopy (FTIR), by determining the cellulose degree of crystallinity by wide angle X-ray scattering (WAXS), by evaluating the mechanical properties, by calculating the activation energy for degradation by thermogravimetric analysis (TGA) and by measuring the optical properties of the Whatman paper samples before and after the treatments setup.

\section{Experimental}

The material studied was Whatman paper in the form of $75 \mathrm{gr} / \mathrm{m}^{2}$, representing high-quality pure cellulose.

In order to obtain model samples with controlled degradation levels, four different accelerating ageing cycles were set up, thus simulating different degradation processes that naturally occur in cellulosic materials.

2.1. Aging Treatments. Thermal oxidation: it was carried out on Whatman samples at $100^{\circ} \mathrm{C}$ in oven for 500 hours in absence of light. It is well known that thermal oxidation induces the breakage of chemical bonds in cellulose and the formation of carbonyl, carboxyl, and hydroperoxide groups [9].

Accelerating ageing in climatic chamber: Whatman samples were kept in a Climatic Chamber Angelantoni Challenge 250E under UV irradiation at a temperature equal to $60^{\circ} \mathrm{C}$ and at a relative humidity of $70 \%$ for 500 hours. Cellulose UV exposure results in the production of free radicals, and in the initiation of chemical reactions including depolymerization, dehydrogenation, dehydroxylation, dehydromethylation, and production of hydrogen, carbon monoxide, and carbon dioxide [10].

Chemical oxidation: it was carried out by dipping Whatman samples in a solution of sodium methaperiodate $\left(\mathrm{NaIO}_{4}\right) 0.1 \mathrm{M}$ for 2 hours. Methaperiodate oxidation is a highly specific reaction and it converts 2,3-dihydroxyl groups in two aldehyde groups, without significant side reactions $[11,12]$.

Photo-oxidation: Whatman samples were exposed in an Angelantoni SB3000E solar box equipped with a Xenon lightsource for 500 hours. Irradiation was kept at a constant power of $1000 \mathrm{~W} / \mathrm{m}^{2}$ and at a temperature of $65^{\circ} \mathrm{C}$. Photooxidative reactions result in an increase of carbonyl content, carboxyl, and hydroperoxide groups [4].

\subsection{Techniques}

2.2.1. Viscosimetric Techniques. The level of molecular degradation of the cellulose constituting the Whatman paper was evaluated by means of a viscosimetric technique improved with respect the standard UNI 8282. In fact, the flow times were measured for cellulose solutions in CED at different dilutions, thus obtaining values of the viscosimetric average polymerisation degree (DPv) of the cellulose more accurate than the DPv values obtained by strictly applying the UNI 8282. The DPv values were measured for cellulose extracted from paper samples before and after the different aging processes undergone.

\subsubsection{Attenuated Total Reflection Fourier Transform Infrared} Analysis (ATR-FTIR). Attenuated total reflection Fourier transform infrared spectroscopy was performed on unaged and aged Whatman samples. The spectra were collected through Jasco FT/IR 6300 spectrometer using singlereflection micro-ATR accessory with diamond ATR element. Spectral resolution was $4 \mathrm{~cm}^{-1}$. The paper samples were previously dried in a desiccator under vacuum at room temperature for 24 hours, in order to eliminate the residual humidity.

2.2.3. Wide Angle X-Ray Scattering Analysis (WAXS). WAXS analysis was carried out on unaged and aged Whatman samples by means of a X'Pert PRO PANalytical diffractometer (Cuk $\alpha \mathrm{Ni}$-filtered radiation) operating in reflection mode. Intensity profiles were collected in the $2 \theta$ range of $5-40^{\circ}$ using a high voltage of $40 \mathrm{kV}$ and a tube current of $40 \mathrm{~mA}$. A standard sample was employed to determine the instrumental broadening.

2.2.4. Mechanical Analysis. Uniaxial tensile tests were carried out on paper samples $(1 \mathrm{~cm} \times 10 \mathrm{~cm})$, by means of an Instron 5564 tensile testing machine operating with a crosshead speed of $10 \mathrm{~mm} / \mathrm{min}$ at the temperature of $25^{\circ} \mathrm{C}$ and $50 \%$ of relative humidity. Before testing, the samples were conditioned at $25^{\circ} \mathrm{C}$ under vacuum for 24 hours. For each sample, both unaged and aged, 10 test pieces were tested, and the results were averaged.

2.2.5. Thermogravimetric Analysis (TGA). The decomposition of the paper samples were investigated by means of thermogravimetric analysis using a Perkin Elmer diamond thermogravimetic/differential thermal analyzer (TG/DTA). Measurements were performed on $10 \mathrm{mg}$ paper samples in an aluminium holder at different heating rates $(1,2.5,5,10$, and $20^{\circ} \mathrm{C} / \mathrm{min}$ ), from $30^{\circ} \mathrm{C}$ to $650^{\circ} \mathrm{C}$ in nitrogen atmosphere in order to determine the thermal stability of the cellulosic materials, related to the different aging process, in terms of the kinetic parameter $E_{A}$ (activation energy).

2.2.6. Colorimetric Analysis. Colorimetric analysis of the unaged and aged paper samples were carried out by means of a spectrophotometer Minolta 3600d in order to determine the variation of aesthetical properties induced by the aging treatments. Color data were calculated under illuminant D65 (daylight $6500^{\circ} \mathrm{K}$ ), 10 standard observers, sphere geometry, specular component included, and UV energy included. The instrument was calibrated against a white standard tile. The colour differences $\left(\Delta E^{*}\right)$ were calculated with the following equation, using the corresponding starting material (unaged paper) as reference:

$$
\Delta E^{*}=\left[\left(\Delta L^{*}\right)^{2}+\left(\Delta a^{*}\right)^{2}+\left(\Delta b^{*}\right)^{2}\right]^{1 / 2},
$$


TABLE 1: DPv values of unaged and aged Whatmann samples.

\begin{tabular}{lc}
\hline Sample & DPv \\
\hline Unaged & $543 \pm 45$ \\
Thermally aged & $427 \pm 12$ \\
Aged in climatic chamber & $206 \pm 2$ \\
Chemically aged & $128 \pm 1$ \\
Photo-oxidated & $86 \pm 3$ \\
\hline
\end{tabular}

where $L^{*}, a^{*}$, and $b^{*}$ are the CIELAB colour space coordinates indicating, respectively, the lightness, the degree of redness and greenness, and the degree of yellowness and blueness.

\section{Results and Discussion}

3.1. Viscosimetric Analysis. The cellulose $\mathrm{DPv}$ values calculated for unaged and aged Whatman paper samples are reported in Table 1. The cellulose extracted from unaged Whatman paper shows a DPv value of $543 \pm 43$, while, according to the expectation, such value decreases for aged samples.

To be noted is that the extent of the observed decrease noticeably depends upon the kind of aging treatment undergone. Thermally oxidised samples exhibit the lowest molecular degradation level with a $\mathrm{DPv}$ value not so far from that calculated for the unaged paper (see Table 1). The lowest $\mathrm{DPv}$ value is conversely exhibited by the photo-oxidated samples, such a value being about six times as low as that shown by the unaged sample. Moreover, chemical oxidation results comparatively more aggressive than climatic chamber aging. Such results suggest a classification of the degradation ability of the treatment carried out: cellulose chains became progressively shorter and shorter starting from samples thermally aged, going through those aged in climatic chamber, then through those chemically aged, up to photo-oxidised samples characterized by the shortest cellulose chains. It ensures that among the aging treatments setup, the photooxidation is the most effective and, thus, dangerous process in inducing cellulose depolymerization.

3.2. FTIR Analysis. Figure 1 compares the ATR-FTIR spectra of Whatman paper unaged (Figure $1(\mathrm{~A})$ ) with those of Whatman papers undergone to the different weathering processes: thermal degradation (Figure 1 (B)), accelerating aging in climatic chamber (Figure $1(\mathrm{C})$ ), chemical degradation (Figure $1(\mathrm{D})$ ), and photo-oxidation (Figure $1(\mathrm{E})$ ).

Generally FTIR analysis of paper samples is complicated owing to the high affinity of the paper with the water. In fact, the band of the absorbed and bonded water is in the region of carbonyl group (between 1635 and $1670 \mathrm{~cm}^{-1}$ ), and sometimes, it can be very broad and can hide the bands of the carbonyl groups. Notwithstanding this, from the region $1500-1800 \mathrm{~cm}^{-1}$ showed in Figure 2, it is possible to observe how different ageing treatments are able to induce different oxidation processes on paper items. In this range, it is in fact possible to detect, for the aged samples, the

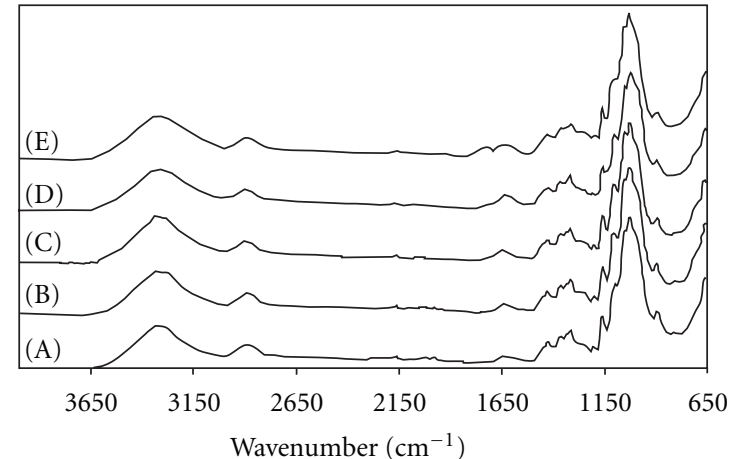

FIGURE 1: FTIR spectra of Whatman paper: (A) unaged; (B) undergone to thermal degradation; $(\mathrm{C})$ undergone to accelerating aging in climatic chamber; (D) undergone to chemical degradation; (E) undergone to photo-oxidation.

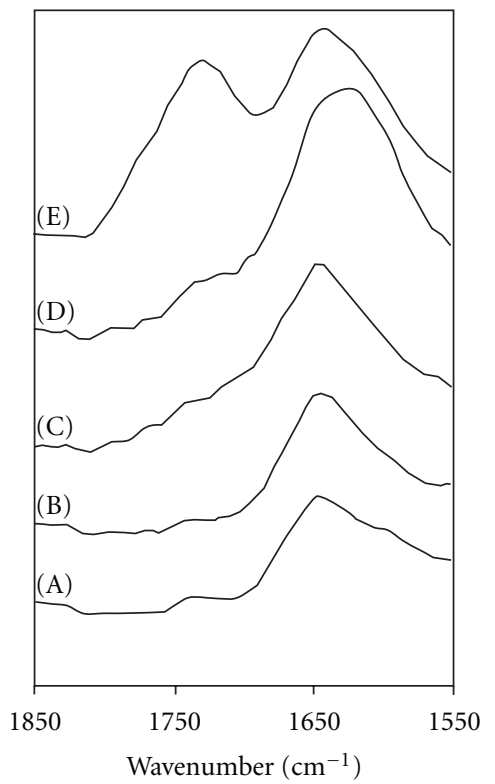

FIgURE 2: FTIR spectra in $1800-1600 \mathrm{~cm}^{-1}$ region of Whatman paper: (A) unaged; (B) undergone to thermal degradation; (C) undergone to accelerating aging in climatic chamber; (D) undergone to chemical degradation; (E) undergone to photo-oxidation.

appearance of a shoulder centered at around $1730 \mathrm{~cm}^{-1}$ due to the carboxyl and/or carbonyl groups. It is interesting to observe that the intensity of such shoulder is a function of the type of weathering treatment: it becomes a distinct band with increasing the depolymerization degree of cellulose, that is, with decreasing the DPv value reported in Table 2, as in the photo-oxidised Whatman (see Figure 2).

Cellulose thermal degradation is expected to cause, besides the scission of chemical bonds between monomeric glucose units, a decrease in its degree of polymerization with the formation of free radicals and carbonyl, carboxyl, and hydroperoxide groups. However, notwithstanding that the performed thermal ageing induces a certain molecular degradation of the samples as observed comparing the $\mathrm{DPv}$ 
TABle 2: Wide angle X-ray diffraction data for unaged and Whatman samples.

\begin{tabular}{lcc}
\hline Sample & Xc $(\%)$ & $1 / \beta_{0}$ \\
\hline Unaged & 73 & 0.46 \\
Thermally aged & 74 & 0.48 \\
Aged in climatic chamber & 78 & 0.51 \\
Chemically aged & 71 & 0.43 \\
Photo-oxidated & 74 & 0.47 \\
\hline
\end{tabular}

values in Table 1, the results of the FTIR measurements, reported in Figures 1 (B) and 2 (B), show that the spectrum of Whatman paper samples thermally aged did not vary significantly in respect with that of unaged paper samples.

Cellulose photo-ageing results in the production of free radicals, and initiation of chemical reactions such as depolymerization, dehydrogenation, dehydroxylation, dehydromethylation, and production of hydrogen carbon monoxide and carbon dioxide. The FTIR spectra of Whatman paper samples aged in climatic chamber (Figure 2 (C)) and photo-oxidated (Figure 2 (E)) show, respectively, a shoulder and a distinct band centered at around $1730 \mathrm{~cm}^{-1}$ due to the carboxyl and/or carbonyl groups. The noticeable increase in the intensities of the absorption band around $1730 \mathrm{~cm}^{-1}$ observed in the FTIR spectrum of photo-oxidated Whatman sample, together with its lowest DPv value measured by means of the viscosimetric analysis, suggests that photodegradation processes occurring on paper sample exposed under a Xenon light source take place mainly by chain scission.

As reported in the literature, the chemical oxidation of cellulose performed with a solution of sodium methaperiodate, a specific oxidising agent, cleaves the C2-C3 bond and involves the formation of a 2,3-dialdehyde following the mechanism of the Malaprade reaction, without significant side reactions [13]. Moreover, it is well known that periodate oxycellulose exhibits two characteristic FTIR bands at near 1740 and $880 \mathrm{~cm}^{-1}$. The absorbance at about $1740 \mathrm{~cm}^{-1}$ is characteristic of carbonyl groups, while the band at around $880 \mathrm{~cm}^{-1}$ is generally assigned to the formation of hemiacetal bonds between the aldehyde groups and neighbour hydroxyl groups. In accordance with the data reported in the literature [13], from the ATR-FTIR spectra of periodate oxidized Whatman paper (Figures 1 (D) and 2 (D)), it can be seen a shoulder centered at around $1730 \mathrm{~cm}^{-1}$ assignable to the $\mathrm{C}=\mathrm{O}$ stretching of carbonyl group, thus aldehydes in nonhydrated form are present.

3.3. Wide Angle X-Ray Scattering Analysis (WAXS). WAXS intensity profiles of Whatman paper samples before and after the artificial aging treatments are shown in Figure 3. As shown, all analaysed samples exhibit the spectrum of cellulose I or "Native" with the characteristic reflections (101), (10) $)$, and (002); the first two are, highly convoluted each other, in the range between 13 and 18 degrees of $2 \theta$ and the third, almost totally resolved very sharp at 22.8 degrees of $2 \theta$. Such results indicate that all the aging treatment

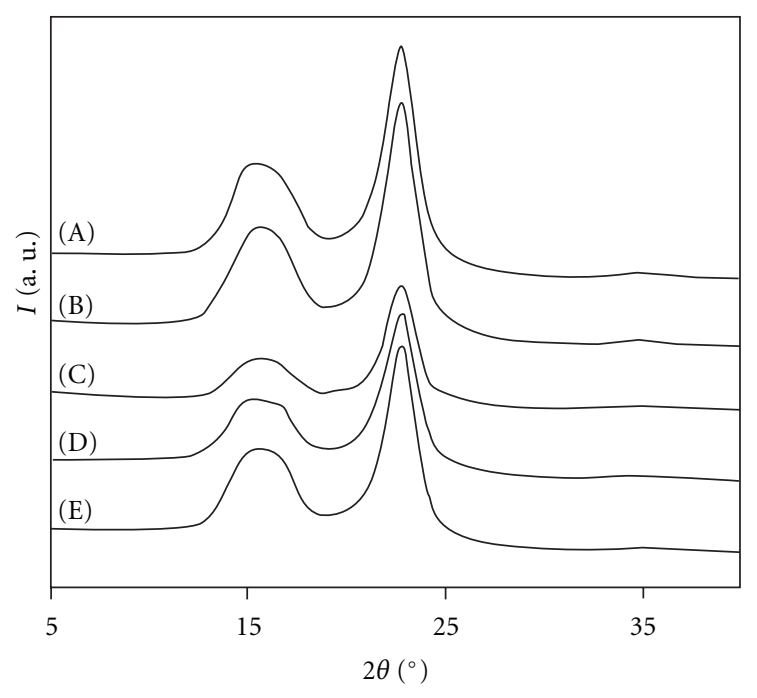

FIGURE 3: WAXS intensity profiles of Whatman paper: (A) unaged; (B) undergone to thermal degradation; (C) undergone to accelerating aging in climatic chamber; (D) undergone to chemical degradation; (E) undergone to photo-oxidation.

performed do not change the cellulose crystalline structure, but all the aged samples still consisting of metastable partially crystalline microfibrils.

Crystallinity index $(X c)$ of cellulose I was calculated according to the Segal method [14] using

$$
X c=\frac{\left(I_{002}-I_{18}\right)}{I_{002}} \times 100 \text {, }
$$

where $X c$ expresses the relative degree of crystallinity, $I_{002}$ is the intensity of the 002 reflection in arbitrary units, and $I_{18}$ is the diffraction intensity in the same unit at $2 \theta=18^{\circ}$, the angle corresponding to the maximum in the WAXS intensity profile of a completely amorphous cellulose.

The crystallinity indexes $(X c)$ calculated for unaged and aged paper samples are reported in Table 2 together the $1 / \beta_{0}$ values for the 002 reflection $\left(1 / A_{002}\right)$ chosen as a relative index to determine cellulose crystal dimension [15]. As shown in Table 2, the $X c$ values are affected by the kind of aging treatments. It is interesting to observe that the thermally oxidized and photo-oxidised samples exhibit the same crystallinity index value (74\%) close to that shown by the unaged paper $(73 \%)$, whereas Whatman samples aged in climatic chamber and chemically oxidized show a $X c$ value respectively higher and lower than that exhibited by the unaged samples. Such findings indicate that there is no direct correlation between chain length and crystallinity (see DPv values reported in Table 1 and $X c$ values reported in Table 2).

The decrease of the amorphous fraction particularly for paper samples aged in climatic chamber could be ascribed to recrystallization and/or to crystal thickening phenomena caused by annealing occurred at constant temperature.

The reduced crystallinity shown by the paper sample deeped in a solution of sodium methaperiodate indicate that periodate easily oxidized cellulose crystalline regions. 


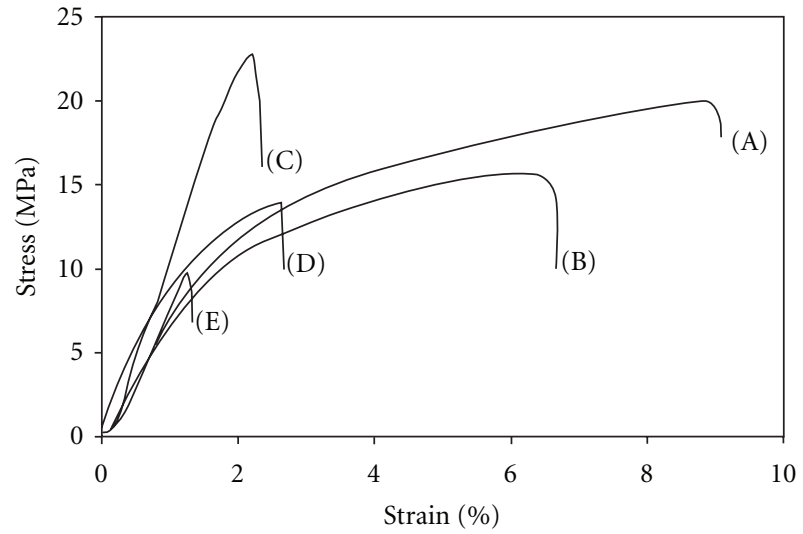

FIgURe 4: Typical stress-strain curves obtained at room temperature for Whatman paper dumbbell-shaped specimens: (A) unaged; (B) undergone to thermal degradation; $(\mathrm{C})$ undergone to accelerating aging in climatic chamber; (D) undergone to chemical degradation; (E) undergone to photo-oxidation.

As shown in Table 2, the cellulose crystal dimensions are in line with $X c$ values and are not strongly affected by the aging process.

3.4. Tensile Behaviour. In Figure 4, typical stress-strain curves obtained at room temperature for Whatman paper dumbbell shaped specimens, unaged and aged, are shown. In Table 3, the results of the mechanical tensile tests carried out on each investigated sample are summarized.

For the unaged paper sample, the Young's modulus value is considerably lower than those exhibited by Whatman paper aged in climatic chamber and photo-oxidised, the highest modulus being shown by the paper samples aged in climatic chamber whose value is more or less twice as much as that evaluated for unaged paper samples. Thermally aged paper samples are characterized by a modulus comparable to that exhibited by the unaged paper, whereas the chemically degraded samples show a comparatively lower tensile modulus. For materials aged in climatic chamber and chemically degraded the modulus performances are to be related to their crystallinity values reported in Table 2. In a first approximation, both crystal buildup (i.e., thickening and perfection) and supermolecular organization (interlamellar amorphous layer thickness and tie molecule density) contribute to determine the change in modulus as a function of crystallinity. Hence, for aged paper samples investigated, the following is to be emphasized: an increased crystallinity induces modulus improvement by a decrease of tie molecules density and vice versa. For thermally and photo-oxidised samples, it is to be also taken into account a cellulose dehydration process occurring at $100^{\circ} \mathrm{C}$ and at $65^{\circ} \mathrm{C}$, respectively, and as consequence an increase of the sample stiffness.

Note, moreover, that the strain at maximum load value, corresponding to strain at break, decreases for all aged paper sample with respect to the unaged sample, thus indicating that all the aging processes greatly influence the stiffness

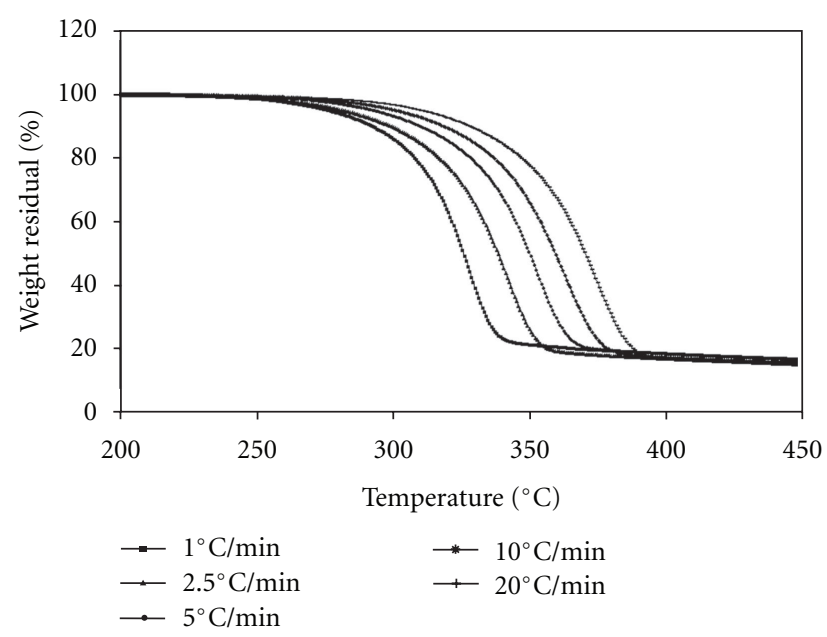

FIGURE 5: Typical degradation curves (\% residual weight against temperature) measured for unaged Whatman sample through TGA at different heating rates between $200^{\circ} \mathrm{C}$ and $450^{\circ} \mathrm{C}$.

of the Whatman paper as show in Table 3, and the strain values tend to decrease with increasing the level of molecular degradation, that is, with decreasing DPv values (see Table 1) and with enhancing the presence of carboxyl and/or carbonyl groups as shown by FTIR analysis.

3.5. Thermogravimetric Analysis (TGA). Thermogravimetric analysis was performed in order to measure the activation energy for the degradation processes that take part through one-step analysis.

Tests were carried out at different heating rates $(1,2.5$, 5 , 10 , and $20^{\circ} \mathrm{C} / \mathrm{min}$ ), from $30^{\circ} \mathrm{C}$ to $650^{\circ} \mathrm{C}$, in nitrogen atmosphere and the kinetic parameter $E_{A}$ (activation energy) was calculated for all the cellulosic materials [16, 17] before and after the different weathering processes.

Typical degradation curves (\% residual weight against temperature) measured for unaged Whatman sample through TGA at different heating rates are reported in Figure 5 in the range $200^{\circ} \mathrm{C}-450^{\circ} \mathrm{C}$; it is evident that the degradation process can be approximated as a single step of weight loss. The first derivative of the curves was calculated for all the samples.

All the Whatman samples are thermally stable until $280^{\circ} \mathrm{C}$; over this temperature, they undergo degradation up to a temperature of approximately $400^{\circ} \mathrm{C}$.

The kinetic values can be obtained using (3) by determining $T_{m}$ (temperature of maximum rate of degradation)

$$
\ln \left[\frac{\partial T / \partial t}{T_{m}^{2}}\right]=\ln \left[k o \frac{R}{E_{A}}\right]-\frac{E_{A}}{R} \cdot \frac{1}{T_{m}},
$$

where $\delta T / \delta t=$ heating rate; $T_{m}=$ temperature of maximum rate of degradation; $k o=$ pre-exponential factor; $R=$ universal gas constant; $E_{A}=$ activation energy of the degradation process.

In agreement with (3), a plot of $\ln \delta T / \delta t * 1 / T_{m}^{2}$ as a function of $1000 / \mathrm{T}\left(\mathrm{K}^{-1}\right)$ should result a straight line whose slope is proportional to the activation energy $E_{A}[18]$. 
TABLE 3: Tensile properties shown by unaged and artificially aged Whatman paper.

\begin{tabular}{lcccc}
\hline Sample & $\begin{array}{c}\text { Maximum load } \\
(\mathrm{N})\end{array}$ & $\begin{array}{c}\text { Strain at max load } \\
(\%)\end{array}$ & $\begin{array}{c}\text { Young's modulus } \\
(\mathrm{MPa})\end{array}$ & $\begin{array}{c}\text { Energy at max load } \\
(\mathrm{mJ})\end{array}$ \\
\hline Unaged & $19.9 \pm 1.0$ & $8.0 \pm 0.5$ & $902 \pm 105$ & $23.4 \pm 2.0$ \\
Thermally aged & $15.5 \pm 0.7$ & $6.3 \pm 0.4$ & $896 \pm 70$ & $14.2 \pm 1.2$ \\
Aged in climatic chamber & $20.5 \pm 1.2$ & $2.0 \pm 0.3$ & $1799 \pm 211$ & $4.5 \pm 0.9$ \\
Chemically aged & $13.3 \pm 1.6$ & $3.1 \pm 0.5$ & $710 \pm 111$ & $4.4 \pm 1.0$ \\
Photo-oxidated & $10.7 \pm 2.3$ & $1.3 \pm 0.1$ & $1302 \pm 184$ & $1.4 \pm 0.4$ \\
\hline
\end{tabular}

TABLE 4: Temeprature of maximum rate of degradation for Whatman paper unaged and undergone to different weathering process.

\begin{tabular}{cccccc}
\hline \multirow{2}{*}{ Heating rate $\left({ }^{\circ} \mathrm{C} / \mathrm{min}\right)$} & \multicolumn{4}{c}{ Temperature of maximum rate of degradation $\left({ }^{\circ} \mathrm{C}\right)$} \\
& Unaged & Thermally aged & Aged in climatic chamber & Chemically aged & Photo-oxidated \\
\hline 1 & 328 & 327 & 324 & 303 & 325 \\
2.5 & 342 & 343 & 337 & 317 & 339 \\
5 & 354 & 353 & 348 & 338 \\
10 & 363 & 365 & 361 & 349 \\
\hline
\end{tabular}

TABLE 5: Kinetic parameter $E_{A}$ (activation energy) for unaged and aged Whatman paper.

\begin{tabular}{lc}
\hline Sample & $E_{A}[\mathrm{~kJ} / \mathrm{mol}]$ \\
\hline Unaged & 208 \\
Thermally aged & 199 \\
Aged in climatic chamber & 195 \\
Chemically aged & 203 \\
Photo-oxidated & 193 \\
\hline
\end{tabular}

The numerical values of the temperature of maximum rate of degradation, $T_{m}$, and the calculated kinetic parameter $E_{A}$ for all the investigated samples are reported in Tables 4 and 5 , respectively.

As shown in Table 5, the sample exhibiting the highest value of the activation energy, and therefore, the best thermal stability is obviously the unaged Whatman paper, while the sample showing the lowest value of $E_{A}$ is the photooxidized paper. The Whatman samples thermally aged, aged in climatic chamber and chemically aged, show intermediate thermal stability.

Hence, for the $E_{A}$ data, it ensures that the photooxidation process is the weathering determining comparatively greater decay of the paper thermal proprieties.

3.6. Evaluation of the Optical Properties. Unaged and aged Whatman samples were submitted to colorimetric analysis.

For the measurement of the optical properties, the $\Delta E^{*}$ value is indicative of the chromatic alteration of paper materials.

In Figure 6, the histogram of $\Delta E^{*}$ values versus the aging undergone are reported for the investigated samples. As shown in Figure 6, the Whatman paper suffers an intense chromatic alteration when subjected to aging in climatic chamber and to photo-oxidation, thus indicating that the exposure to Xenon arc lamp and to UV light in presence

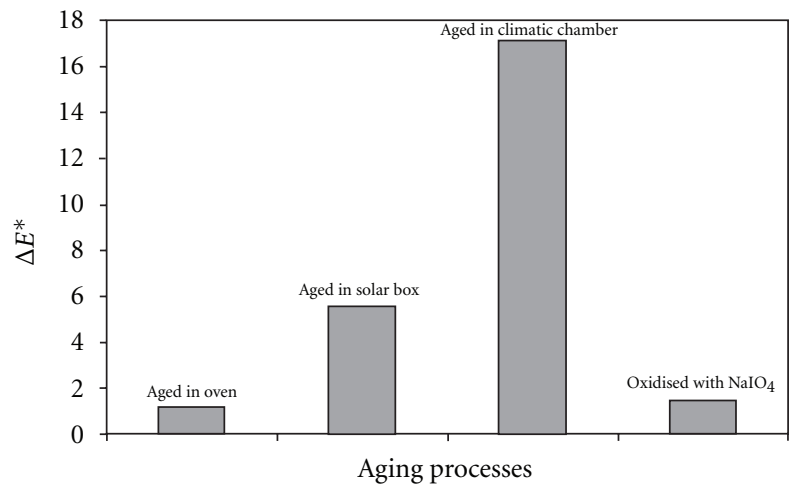

FIgURE 6: Histogram of $\Delta E^{*}$ versus the different aging processes.

of moisture promotes the formation of chromophores. Moreover, the obtained results indicate that the chromatic alteration induced by chemical oxidation is comparatively less intense than that induced by thermal aging. Considering the FTIR results previously discussed, it can be hypothesised that the extend of chromatic alteration increase with increasing the formation of carboxyl and/or carbonyl compounds as revealed by the appearance of a new shoulder in the FTIR spectra with a relative maximum at $1730 \mathrm{~cm}^{-1}$ (see Figure 2). The finding that paper samples aged in climatic chamber shows chromatic alteration comparatively higher than that exhibited by the photo-oxidized samples notwithstanding a lower presence of carbonyl/carboxyl compounds could be ascribed to the presence of moisture also contributing to the formation of chromophores containing degradation products.

\section{Concluding Remarks}

With the aim at determining the physicochemical, mechanical, and aesthetical alterations induced by four different 
artificial aging treatments performed on Whatman paper samples, the cellulose degradation was investigated by means of viscosimetric, FTIR, WAXS, and TGA techniques together with mechanical and colorimetric analyses. It was found that all the accelerated aging processes set up significantly modify the structure and the properties of the Whatman paper, the extent of the deterioration effects undergone depending upon the type of treatment carried out. Thus, to prevent paper exposed to different environmental conditions, the following is to be taken into account.

Cellulose dehydration only induced by temperature produces a decrease in strain at break and consequently paper stiffness, notwithstanding that thermally aged samples show a low level of molecular depolymerization and of carbonyl/carboxyl formation.

Simultaneous effects of temperature and light cause conversely high molecular degradation with a presence of carboxyl and/or carbonyl groups strongly increased. Analogous results were obtained for Whatman paper samples chemically aged with sodium methaperiodate.

To be underlined that with decreasing the cellulose DPv value, a new absorption band appears in the FTIR spectra due to the carboxyl and/or carbonyl groups, indicating a direct correlation between chain length and carboxyl and/or carbonyl groups presence. Also, the cellulose strain at break was found correlated with the chain length, strain at break values decreasing with decreasing $\mathrm{DPv}$ values. The specific oxidation of by periodic acid, which cleaves the C2-C3 bond in the glucosidic ring and converts the 2,3-dihydroxyl groups in two aldheyde groups is the only aging which induces a reduction in cellulose crystallinity. The different Young's modulus performances shown by the aged paper were found to be mainly due to crystallinity for paper aged in climatic chamber and chemically treated and to cellulose dehydration for thermally aged and photo-oxidised paper. Remarkable yellowing phenomena on paper surface are, moreover, produced by combined effects of temperature and light enhanced by moisture presence.

\section{Acknowledgments}

The authors are thankful to Dr. Elisabetta Princi and Silvia Vicini from the University of Genova, Department of Chemistry and Industrial Chemistry, for providing Whatman paper samples chemically aged. Thanks are also due to CRdC INNOVA for FTIR and WAXS investigation.

\section{References}

[1] M. Paci, C. Federici, D. Capitani, N. Perenze, and A. L. Segre, "NMR study of paper," Carbohydrate Polymers, vol. 26, no. 4, pp. 289-297, 1995.

[2] G. A. F. Roberts and J. C. Roberts, Eds., Paper Chemistry, Chapman and Hall, Glasgow, UK, 1991.

[3] R. L. Feller, Accelerated Aging Photochemical and Thermal Aspects, The Getty Conservation Institute, 1994.

[4] J. Malešič, J. Kolar, M. Strlič et al., "Photo-induced degradation of cellulose," Polymer Degradation and Stability, vol. 89, no. 1, pp. 64-69, 2005.
[5] J. Kolar, M. Strlic, S. Pentzien, and W. Kautek, "Near-UV, visible and IR pulsed laser light interaction with cellulose," Applied Physics A, vol. 71, no. 1, pp. 87-90, 2000.

[6] S. Soares, N. M. P. S. Ricardo, S. Jones, and F. Heatley, "High temperature thermal degradation of cellulose in air studied using FTIR and $\mathrm{H}$ and C solid-state NMR," European Polymer Journal, vol. 37, no. 4, pp. 737-745, 2001.

[7] P. M. Whitmore and J. Bogaard, "The effect of oxidation on the subsequent oven aging of filter paper," Restaurator, vol. 16, no. 1, pp. 10-30, 1995.

[8] S. Soares, G. Camino, and S. Levchik, "Comparative study of the thermal decomposition of pure cellulose and pulp paper," Polymer Degradation and Stability, vol. 49, no. 2, pp. 275-283, 1995.

[9] J. Rychlý, L. Matisová-Rychlá, M. Lazár et al., "Thermal oxidation of cellulose investigated by chemiluminescence. The effect of water at temperatures above $100{ }^{\circ} \mathrm{C}$," Carbohydrate Polymers, vol. 58, no. 3, pp. 301-309, 2004.

[10] N. S. Hon, "Fundamental degradation processes relevant to solar irradiation of cellulose: ESR studies," Journal of Macromolecular Science: Pure and Applied Chemistry, vol. 10, no. 6, pp. 1175-1192, 1976.

[11] U. J. Kim, S. Kuga, M. Wada, T. Okano, and T. Kondo, "Periodate oxidation of crystalline cellulose," Biomacromolecules, vol. 1, no. 3, pp. 488-492, 2000.

[12] S. Vicini, E. Princi, G. Luciano et al., "Thermal analysis and characterisation of cellulose oxidised with sodium methaperiodate," Thermochimica Acta, vol. 418, no. 1-2, pp. 123-130, 2004.

[13] Q. G. Fan, D. M. Lewis, and K. N. Tapley, "Characterization of cellulose aldehyde using Fourier transform infrared spectroscopy," Journal of Applied Polymer Science, vol. 82, no. 5, pp. 1047-1305, 2001.

[14] L. Segal, J. J. Creely, A. E. Martin, and C. M. Conrad Jr., "An empirical method for estimating the degree of crystallinity of native cellulose using the X-ray diffractometer," Textile Research Journal, vol. 29, pp. 786-794, 1959.

[15] E. Princi, S. Vicini, E. Pedemonte et al., "Physical and chemical characterization of cellulose based textiles modified by periodate oxidation," Macromolecular Symposia, vol. 218, pp. 343-352, 2004.

[16] M. Cocca, L. D’Arienzo, L. D’Orazio, G. Gentile, and E. Martuscelli, "Polyacrylates for conservation: chemico-physical properties and durability of different commercial products," Polymer Testing, vol. 23, no. 3, pp. 333-342, 2004.

[17] P. Nising, T. Zeilmann, and T. Meyer, "On the degradation and stabilization of poly (methyl methacrylate) in a continuous process," Chemical Engineering and Technology, vol. 26, no. 5, pp. 599-604, 2003.

[18] T. Kashiwagi and T. Hirata, "Thermal and oxidative degradation of poly(methyl methacrylate): molecular weight," Macromolecules, vol. 18, no. 2, pp. 131-138, 1985. 

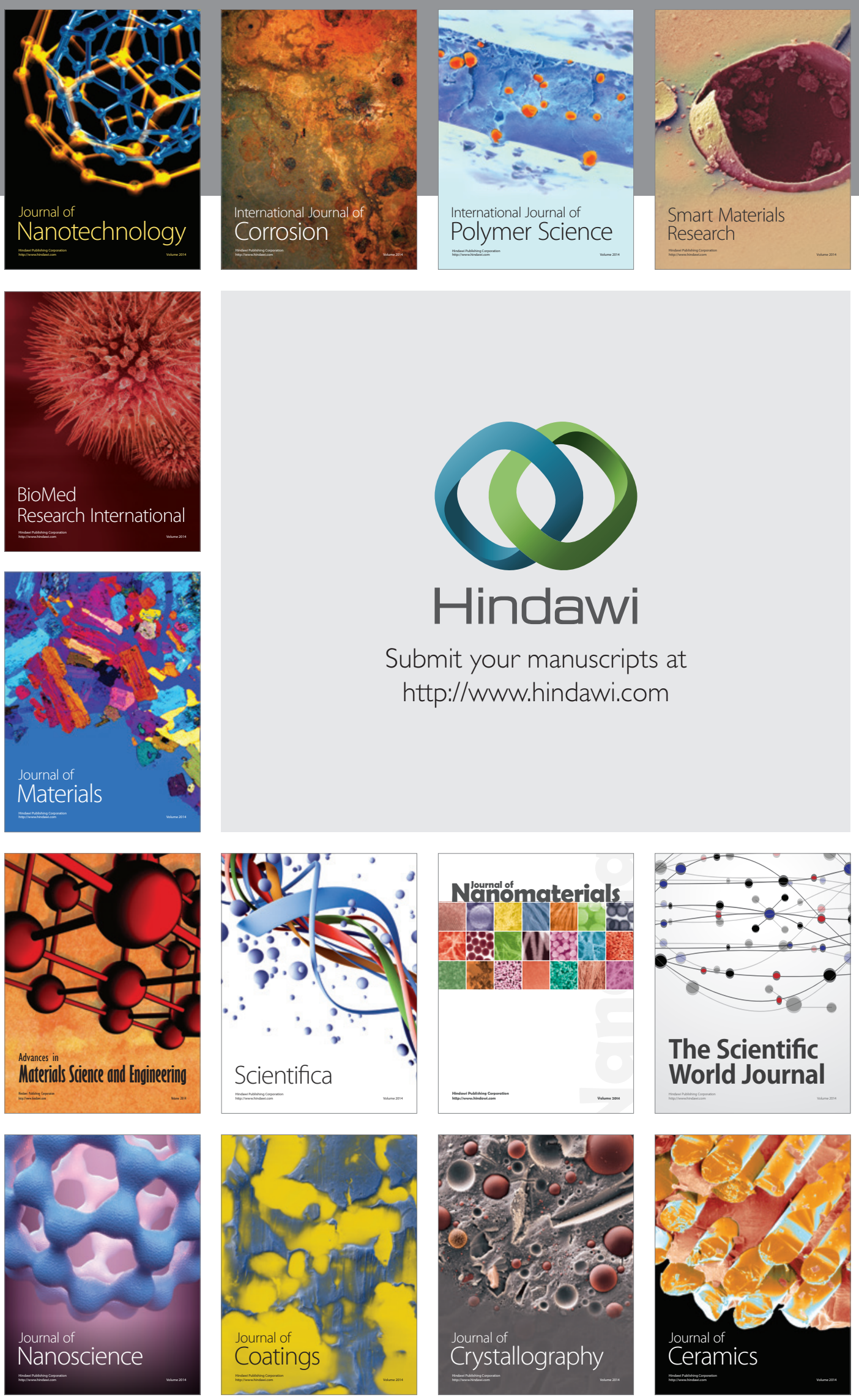

The Scientific World Journal

Submit your manuscripts at

http://www.hindawi.com

\section{World Journal}

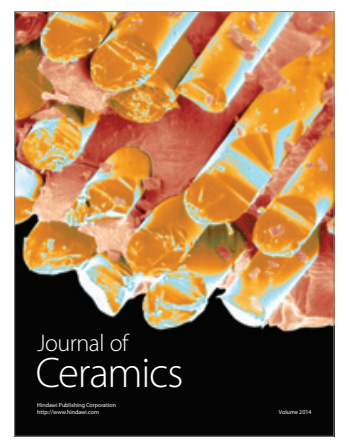

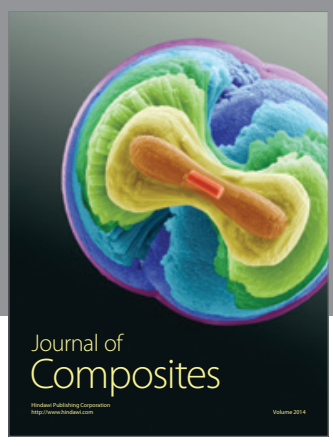
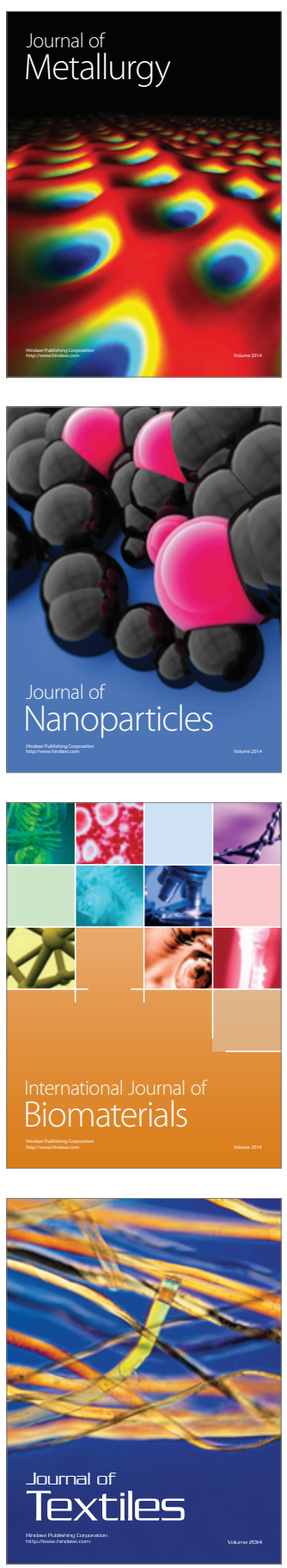